\title{
Psicología
}

Artículo Original

\section{Prevalencia y factores asociados al trastorno de estrés postraumático en personas desplazadas en Colombia}

\section{Prevalence and factors associated with post-traumatic stress disorder in displaced persons in Colombia}

Morales Mesa Santiago Alberto ${ }^{1 凶} \underline{\mathrm{ORCID}}$; Agudelo Martínez Alejandra María $2 \underline{\square R C I D}$; Dedsy Yajaira Berbesi Fernández ${ }^{3 凶} \underline{\mathrm{ORCID}}$

\footnotetext{
1 Magister en Salud Pública, Magister en Epidemiología. Docente Facultad de Psicología y Enfermería. Grupos de investigación: Cuidado Enfermería CES y Psicología, Salud y Sociedad. Universidad CES, Medellín, Colombia.

${ }^{2}$ Magister en Salud Pública, Magister en Epidemiología. Docente Facultad de Ciencias de la Nutrición y los Alimentos. Grupos de investigación: Nutral, Salud Mental y Epidemiología y Bioestadística. Universidad CES.

${ }^{3}$ Doctora en Epidemiología y Bioestadística. Decana y Docente Facultad de Enfermería, Grupo de investigación: Cuidado Enfermería CES, Universidad CES.
}

Fecha correspondencia: Recibido: abril 2 de 2020. Aceptado: julio 30 de 2021.

Forma de citar:

Morales, S., Agudelo, A., \& Berbesi, D. (2021). Prevalencia y factores asociados al trastorno de estrés postraumático en personas desplazadas en Colombia. Rev. CES Psico, 14(3), 134-150. https://dx.doi.org/10.21615/ cesp. 5448

Open access

(C) Derecho de autor Licencia creative commons Ética de publicaciones Revisión por pares Gestión por Open Journal System DOI: $10.21615 /$ cesp.5448 ISSNe: $2011-3080$

Publica con nosotros

\section{Resumen}

Introducción: el trastorno por estrés postraumático (TEPT) es un síndrome que sobreviene después de que una persona experimenta un acontecimiento traumático extremo como la violencia en sus diversas manifestaciones: doméstica, sexual, política o armada. Objetivo: determinar la prevalencia del TEPT, los factores demográficos y las condiciones del desplazamiento asociadas en población desplazada por la violencia producto del conflicto armado, en tres ciudades de Colombia: Bogotá, Medellín y Buenaventura. Método: estudio observacional, analítico con diseño cross-sectional, medición transversal, mediante disponibilidad de datos retrospectivos. La población objeto de estudio se compone de 1026 registros de personas víctimas del conflicto armado, con edades entre 13 y 65 años, participantes del estudio primario "Salud mental en víctimas de desplazamiento forzado por la violencia en Colombia. El caso de Bogotá, Medellín y Buenaventura". Resultados: la prevalencia de vida del TEPT es de 9,9\%, siendo superior en la ciudad de Buenaventura; el trastorno presenta una asociación estadísticamente significativa 
con variables como la ocupación, el estado civil y la forma como se llevó a cabo el desplazamiento. Conclusiones: resaltan aspectos como la falta de ocupación, las características del desplazamiento y el bajo apoyo social como posibles factores que desencadenan o intensifican el TEPT.

Palabras clave: prevalencia; trastornos por estrés postraumático; violencia social; personas desplazadas.

\section{Abstract}

Introduction: post-traumatic stress disorder (PTSD) is a syndrome that occurs after a person experiences an extreme traumatic event such as violence in its various manifestations: domestic, sexual, political, or armed. Objective: to determine the prevalence of PTSD and the demographic factors and associated displacement conditions in the population displaced by the violence resulting from the armed conflict, in three cities of Colombia: Bogotá, Medellín and Buenaventura. Method: observational, analytical study with cross-sectional design, crosssectional measurement, through availability of retrospective data. The population under study consists of 1,026 records of people who are victims of the armed conflict, aged between 13 and 65 years, participants in the primary study "Mental disorders in displaced population victims of the armed conflict, in three cities of Colombia". Results: the lifetime prevalence of PTSD is 9.9\%, higher in the city of Buenaventura; the disorder presents a statistically significant association with variables such as occupation, marital status, and the way in which the displacement occurred. Conclusions: aspects such as lack of job, displacement characteristics and low social support stand out as possible factors that trigger or intensify PTSD.

Keywords: prevalence; post-traumatic stress disorders; social violence; displaced people.

\section{Introducción}

El trastorno por estrés postraumático (TEPT) es un síndrome que sobreviene después de que una persona presencia o experimenta un acontecimiento traumático extremo, y en respuesta presenta una tríada sintomática de fenómenos invasores, conductas de evitación y síntomas de hipervigilancia que pueden presentarse a cualquier edad (Achilli, Rodriguez, \& Folino, 2014; Ehlers, Hackmann, \& Michael, 2004).

En cuanto a sus condiciones, el acontecimiento traumático puede durar segundos, horas, días o meses; ocurrir en forma única (accidente de tránsito) o reiterada (combate, maltrato infantil intrafamiliar); tener consecuencias físicas, psicológicas y sociales tanto en el ámbito individual 
como en el colectivo, y originar diversas manifestaciones clínicas que van desde síntomas emocionales aislados hasta un cuadro psicótico (Beck \& Sloan, 2012; Ozer, Best, Lipsey, \& Weiss, 2003).

En las últimas décadas, ha crecido el interés por conocer la predisposición para presentar este trastorno en población desplazada por la violencia. El desplazamiento forzado es una problemática que ha estado presente en la historia de la humanidad y que se genera como producto de trasformaciones, cambios y en algunos casos conflictos sociales, económicos y políticos. En Colombia, el desplazamiento forzado se ha derivado principalmente del conflicto interno armado, producto del enfrentamiento permanente entre grupos al margen de la ley, como la guerrilla y los paramilitares, y el ejército (Echenique, Medina, Medina, \& Ramírez, 2008; Sarmiento, 2016; Morales, Muñoz, Ghiso, Tobón, \& Patiño, 2009).

Para el año 2019, la Agencia de la ONU para los refugiados -ACNUR-en su informe de tendencias mundiales, afirmó que Colombia registró el mayor número de personas desplazadas internamente; de este modo, y de acuerdo con la Red Nacional de Información -RNI- (2020), desde 1985 al 2019 se acumularon cerca de ocho millones de casos, lo que evidencia la magnitud del problema. Según la RNI, en el 2019, en Colombia, 74.772 personas fueron expulsadas de su lugar de origen; Antioquia registró 12.760 personas y Valle del Cauca 10.442, siendo dos de los departamentos más afectados en el país.

Como consecuencia de los enfrentamientos y del desplazamiento forzado, la estabilidad económica, social y cultural de Colombia y, la salud mental de muchos de sus habitantes se ha visto afectada, en especial en algunas regiones rurales donde la población ha sufrido con más agudeza estas situaciones (Ferrel, Ferrel, Cañas-Herazo, Barros, \& Yañez, 2020); siendo el TEPT una de las afectaciones más frecuentes (Diaz \& Serrano, 2016).

En Colombia, los hallazgos del Estudio Nacional de Salud Mental (2015) reportan, en lo que respecta al TEPT en población general, una prevalencia global en la vida entre 3\% y $8 \%$, siendo mayor en mujeres que en hombres, en comparación a un 10\% - 15\% de prevalencia de síntomas de TEPT en población desplazada, siendo también las mujeres el grupo poblacional que presenta las más altas prevalencias (Torres-Salazar, Mejía-Jaimes, Conde-Cotes, \& Botelho-De Oliveira, 2021). 
Los daños mentales que se producen en poblaciones de desplazados por la violencia han sido determinados a través de su prevalencia, y de consecuencias y síntomas psicológicos como reviviscencias del evento, ansiedad, hipervigilancia, alteración emocional que incide en el resto del funcionamiento psicosocial, conflictos en las relaciones interpersonales, dificultades en el cumplimiento de tareas habituales, entre otros (Ministerio de Salud y Protección Social \& Colciencias, 2015).

Algunos estudios revelan que la prevalencia global del TEPT oscila entre el 1 y $14 \%$, explicándose esta variabilidad por los criterios diagnósticos empleados y el tipo de población objeto de estudio. En investigaciones realizadas con grupos de individuos que viven en condiciones de mayor riesgo para presentar TEPT se encuentran cifras de prevalencia que van del 3 al 58\% (Bados, 2015; Ministerio de la Protección Social de Colombia, Oficina de las Naciones Unidas contra la Droga y el Delito [UNODC], \& Universidad CES, 2010). En países como Argelia, Camboya, Buthan y Nepal, la prevalencia del TEPT es de $37 \%, 28 \%$, $43 \%$ y $25 \%$, respectivamente (Campo-Arias, Oviedo, \& Herazo, 2014; Alejo, Rueda, Ortega, \& Orozco, 2007; Bados, 2015).

Teniendo en cuenta esta realidad en el ámbito nacional e internacional, y la importancia y necesidad de identificar los factores de riesgo y protección asociados al TEPT en población desplazada en Colombia para la implementación de programas de promoción de la salud mental, el presente estudio tuvo como objetivo determinar la prevalencia mes, último año y vida y los factores asociados al TEPT en población en situación de desplazamiento por la violencia, en tres ciudades de Colombia: Bogotá, Medellín y Buenaventura.

\section{Método}

Se realizó un estudio observacional, con diseño cross-sectional e intención analítica, apoyado en datos retrospectivos de la población desplazada víctima de la violencia de las ciudades de Bogotá, Medellín y Buenaventura, Colombia, con y sin TEPT.

La información se tomó de la base de datos del estudio "Salud mental en víctimas de desplazamiento forzado por la violencia en Colombia. El caso de Bogotá, Medellín y Buenaventura", desarrollado por el Grupo de Salud Mental de la Universidad CES (2018) y financiado por Colciencias en el marco de la convocatoria 711-2015. Este estudio utilizó como instrumento de recolección de datos la encuesta internacional diagnostica de trastornos mentales (Composite International Diagnostic Interview -CIDI-Versión CAPI- OMS), la cual ha sido seleccionada como instrumento oficial que se aplica en el Estudio Mundial de Salud 
Mental. Esta encuesta contiene un tamizaje que determina los módulos a preguntar al entrevistado de entre 22 secciones diagnósticas que valoran diferentes trastornos mentales, como los de ansiedad, por abuso de sustancias, del estado del ánimo, la planeación e intento suicida y el TEPT. Para indagar por aspectos relacionados con el desplazamiento, en el estudio primario se construyó un instrumento "ad hoc" con preguntas sobre las condiciones del desplazamiento y del asentamiento, las cuales fueron respondidas mediante un aplicativo construido en Access.

Las variables analizadas en la presente investigación son retomadas del estudio "Salud mental en víctimas de desplazamiento forzado por la violencia en Colombia. El caso de Bogotá, Medellín y Buenaventura", y se agrupan en: sociodemográficas y condiciones del desplazamiento, las cuales se relacionan con la variable TEPT, y su prevalencia de vida, anual y en el último mes.

La población objeto de estudio estuvo compuesta por 1.026 registros de la población víctima de conflicto armado, con edades entre 13 y 65 años, obtenidos del estudio primario; se buscó que fuera representativo y proporcional para cada ciudad (33\%). En lo referente a la selección de las personas desplazadas víctimas del conflicto armado, el estudio primario utilizó un muestreo probabilístico, multietapico, estratificado (centros de atención a víctimas de cada ciudad) de forma sistemática, garantizando que cada persona víctima de desplazamiento forzado tuviera la misma probabilidad de ser seleccionada. Las personas que aceptaron hacer parte de la investigación confirmaron su participación por medio del respectivo consentimiento o asentimiento informado de acuerdo con su edad.

El proyecto fue avalado por el Comité de Ética de la Universidad CES, se realizó única y exclusivamente con fines académicos, respetando en todo momento la privacidad y confidencialidad de las personas. Esta investigación es considerada sin riesgo de acuerdo con los criterios establecidos en la resolución 8430 de 1993 del Ministerio de Salud y Protección Social de Colombia.

Se incluyeron todos los registros de la base de datos del estudio primario y se utilizó el paquete estadístico SPSS licencia 21 (Universidad CES) para el procesamiento de la información. LoS análisis de los datos se realizaron a través de distribuciones de frecuencias; con el fin de determinar los factores asociados se implementó la prueba Chi-cuadrado de independencia de Pearson, además se utilizó como medida epidemiológica el Odds Ratio (OR) con sus respectivos intervalos de confianza del 95\% y se utilizó como nivel de significación un valor del 5\%. 


\section{Resultados}

Con respecto a la distribución del total de la muestra, según el sexo y la edad, los datos son muy homogéneos, casi en una relación de 1 a 1 entre hombres y mujeres; en cuanto a la edad, los grupos de 18 a 29 y 30 a 44 tuvieron igual representación con el 27,5 \% cada uno, lo que equivale a un poco más de la mitad población.

Es de resaltar que de cada 10 encuestados $(91,1 \%)$, nueve vivían en estratos socioeconómicos bajos. En relación con la escolaridad, por cada dos personas afectadas por la violencia y el desplazamiento forzado, uno alcanzó el nivel de secundaria, en tanto el 40,5\% no tiene estudios o solo completó el nivel de primaria incompleta. El estado civil que más predominó fue soltero, seguido de unión libre, representado por el $36.0 \%$.

La ocupación que más predominó fue la de trabajador, es decir, aquellos que ejercían una actividad de la cual devengaban un salario para su sostenimiento con el 32,1 \%; no obstante, cuando se hace un comparativo con el régimen de seguridad social al que están adscritos, solo $17,8 \%$ refirió ser cotizante, es decir, que por cada dos personas que manifiestan estar laborando solo una aporta al sistema de Salud y Seguridad Social del país que es un deber al tener condición de trabajador. Se destaca que nueve de cada cien personas que hicieron parte del estudio no tienen ningún tipo de seguridad social (ver Tabla 1).

Con respecto a las condiciones en las que se presentó el desplazamiento, el más común fue el que se llevó a cabo con otras personas, equivalente a 88,4 \% y el 91,6\% expresó que su desplazamiento se llevó a cabo hace más de un año.

En cuanto al actor que influyó en el desplazamiento, los encuestados expresaron en primera instancia que fue la guerrilla (58,1\%), seguida de los paramilitares (33,6 \%). El 87,0 \% de la población de estudio indicó que su desplazamiento se realizó de forma intempestiva; 44,2\% salieron caminando y $44,5 \%$ utilizando un transporte público (ver Tabla 2). 
Tabla 1. Distribución porcentual de los factores demográficos en la población de estudio ( $\mathrm{N=1} 026)$.

\begin{tabular}{|c|c|c|}
\hline Factores demográficos & Categorías & $\begin{array}{l}\text { Frecuencia relativa\% } \\
\qquad(n=1026)\end{array}$ \\
\hline \multirow{2}{*}{ Sexo } & Hombre & 46,5 \\
\hline & Mujer & 53,5 \\
\hline \multirow{4}{*}{ Grupos de edad (años) } & 13 a 17 & 20,5 \\
\hline & 18 a 29 & 27,5 \\
\hline & 30 a 44 & 27,5 \\
\hline & 45 a 65 & 24,6 \\
\hline \multirow{4}{*}{ Estrato socioeconómico } & Sin estrato & 3,4 \\
\hline & Bajo & 91,1 \\
\hline & Medio & 2,3 \\
\hline & No sabe & 3,1 \\
\hline \multirow{4}{*}{ Régimen de seguridad social } & Contributivo & 17,8 \\
\hline & Subsidiado & 71,9 \\
\hline & No asegurado & 9,4 \\
\hline & Otro & 0,9 \\
\hline \multirow{5}{*}{ Nivel de escolaridad } & Sin estudios & 6,1 \\
\hline & Primaria incompleta & 34,4 \\
\hline & Secundaria & 50,6 \\
\hline & Técnico/tecnólogo & 5,9 \\
\hline & Universitaria & 2,9 \\
\hline \multirow{5}{*}{ Ocupación } & Trabaja & 32,1 \\
\hline & Estudiante & 21,6 \\
\hline & $\begin{array}{l}\text { No trabaja ni busca } \\
\text { trabajo }\end{array}$ & 5,3 \\
\hline & Ama de casa & 25,1 \\
\hline & Otra & 15,9 \\
\hline \multirow{6}{*}{ Estado civil } & Soltero & 47,4 \\
\hline & Casado & 8,5 \\
\hline & Unión libre & 36,0 \\
\hline & Separado/divorciado & 6,0 \\
\hline & Viudo & 2,1 \\
\hline & Otros & 4,4 \\
\hline \multirow{3}{*}{ Ciudad } & Bogotá & 33,8 \\
\hline & Buenaventura & 33,3 \\
\hline & Medellín & 32,9 \\
\hline
\end{tabular}


Tabla 2. Distribución porcentual de las condiciones del desplazamiento en la población de estudio ( $\mathrm{N}=1026)$.

\begin{tabular}{llc}
\hline Condiciones del desplazamiento & \multicolumn{1}{c}{ Categorías } & $\begin{array}{c}\text { Frecuencia relativa\% } \\
\text { (n=1026) }\end{array}$ \\
\hline \multirow{2}{*}{ Desplazamiento individual } & $\mathrm{Si}$ & 11,6 \\
\cline { 2 - 3 } Tiempo de desplazado & No & 88,4 \\
\hline \multirow{2}{*}{ Número de desplazamientos } & Un año o menos & 8,4 \\
\cline { 2 - 3 } & Más de un año & 91,6 \\
\hline \multirow{2}{*}{ Actor que influyó en el } & Uno & 80,8 \\
\cline { 2 - 3 } desplazamiento & Dos o más & 19,2 \\
& Guerrilla & 58,1 \\
\hline \multirow{2}{*}{ Desplazamiento intempestivo } & Paramilitares & 33,6 \\
\hline \multirow{2}{*}{$\begin{array}{l}\text { Forma en que sale del lugar de } \\
\text { residencia en el desplazamiento }\end{array}$} & Delincuencia común & 3,2 \\
\cline { 2 - 3 } & Ejercito & 0,7 \\
\cline { 2 - 3 } & Otros & 4,4 \\
\cline { 2 - 3 } & Si & 87,0 \\
\cline { 2 - 3 } & Caminando & 13,0 \\
\cline { 2 - 3 } & Servicio público & 44,2 \\
\hline
\end{tabular}

De acuerdo con el comportamiento de la prevalencia de vida del TEPT por ciudades, se encontró que la de mayor peso porcentual es Buenaventura con el 11,4 \%, seguida de Bogotá con 9,8 \% y por último Medellín con 8,6 \%.

La prevalencia de año del TEPT conservó el mismo patrón; no obstante, la prevalencia de TEPT en el último mes, es muy similar en todas las ciudades: Buenaventura 4,7\%, Bogotá 4,3\% y Medellín 4,2 \%.

La prevalencia de vida del TEPT está representada en un 9,9\%, es decir, que de cada diez personas que hicieron parte de la muestra una de ellas ha presentado este trastorno en algún momento de su vida hasta el período que fue encuestado, mostrando una distribución por sexo superior en mujeres y en adultos (ver Gráfico 1). La prevalencia de TEPT en el último año disminuye un poco respecto a la de vida, representada en el 7,3 \%, y la prevalencia en el último mes, disminuye más de la mitad en comparación con la anual; evidenciado que solo 4,4 de cada cien han presentado el trastorno en el último mes. 

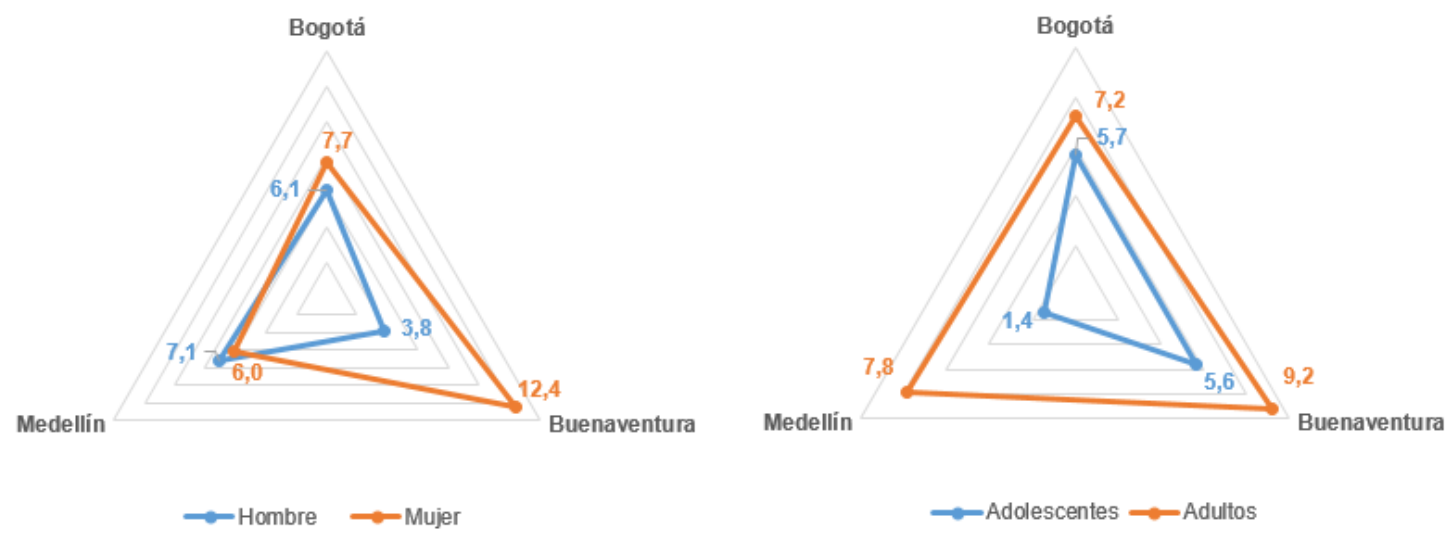

Gráfico 1. Prevalencia anual del Trastorno de estrés postraumático según sexo y grupo de edad en tres ciudades de Colombia.

Aunque no existe una asociación estadísticamente significativa $X^{2}(p=0,058)$, los resultados muestran que del total de personas que padecen TEPT, $64 \%$ eran mujeres y el $36 \%$ restante hombres; es decir, que ser mujer aumenta las probabilidades de presentar TEPT (OR= $1,57995 \%$ IC 0,980-2,602).

Con respecto a la edad, tampoco se presentó una asociación estadísticamente significativa $X^{2}(p=0,084)$, sin embargo, el grupo de personas de mayor edad tiene dos veces más de probabilidad de presentar TEPT con respecto a los de más baja edad (OR=2,569 IC95 \% 1,176 $5,613)$.

Las únicas variables de los factores sociodemográficos que presentaron asociación estadísticamente significativa con el TEPT fueron la ocupación $X^{2}(p=0,012)$ y el estado civil $X^{2}$ $(p=0,037)$. Las amas de casa y quienes están trabajando presentaron mayor peso porcentual ( $38,7 \%$ y $25,3 \%$, respectivamente); y la probabilidad de presentar TEPT es dos veces mayor en quienes no trabajan ni buscan trabajo que en quienes se dedican a una actividad económica (OR=2.066 IC95 \% 1,130 - 3,777, p= 0,012). Además, los estudiantes tienen dos veces más probabilidad de presentar TEPT respecto a quienes se dedican a otra actividad, aunque no es una relación estadísticamente significativa (OR=2,039 IC \% 0,776- 5,363).

Las personas viudas tienen una probabilidad de aproximadamente cinco veces más de presentar TEPT en comparación con los solteros, presentando una significancia de $p=(0,037)$ (OR= 4,811 IC95 \% 1,654 - 13,993); igualmente, quienes están separados o divorciados tienen 1,7 veces más la probabilidad de presentar TEPT en comparación con los solteros, aunque no existe significancia estadística (OR= 1,753 IC95 \% 0,695 - 4,417). 
Si bien no se encontró asociación entre el TEPT y el nivel de escolaridad, 48 \% de quienes presentaron el trastorno tenían grado de primaria, y 38,7 \% grado bachillerato. $Y$ tener primaria incompleta aumenta 1,5 veces la probabilidad de presentar TEPT frente al quienes presentan nivel universitario (OR= 1,590 IC95 \% 0,364 - 6,952) (ver Tabla 3).

Tabla 3. Asociación entre los factores demográficos y el trastorno por estrés postraumático ( $\mathrm{N}=1026)$.

\begin{tabular}{|c|c|c|c|c|c|c|c|}
\hline \multirow{2}{*}{\multicolumn{2}{|c|}{ Factores demográficos }} & \multicolumn{2}{|c|}{ Si } & \multicolumn{2}{|c|}{ No } & \multirow[t]{2}{*}{ Valor $p$} & \multirow[t]{2}{*}{ OR } \\
\hline & & $n$ & $\%$ & $n$ & $\%$ & & \\
\hline \multirow{2}{*}{ Sexo } & Hombre & 27 & 36,0 & 450 & 47,3 & 0,058 & 1 \\
\hline & Mujer & 48 & 64,0 & 501 & 52,7 & & $1,579(0,980-2,602)$ \\
\hline \multirow{4}{*}{ Grupos de edad } & 13 a 17 años & 9 & 12,0 & 201 & 21,1 & 0,084 & 1 \\
\hline & 18 a 29 años & 22 & 29,3 & 260 & 27,3 & & $1,890(0,852-4,193)$ \\
\hline & 30 a 44 años & 18 & 24,0 & 264 & 27,8 & & $1,523(0,670-3,461)$ \\
\hline & 45 a 65 años & 26 & 34,7 & 226 & 23,8 & & $2,569(1,176-5,613)$ \\
\hline \multirow{3}{*}{ Estrato socioeconómico } & Sin estrato & 2 & 2,7 & 33 & 3,5 & 0,902 & $1,335(0,119-16,296)$ \\
\hline & Bajo & 70 & 93,3 & 865 & 91,0 & & $1,896(0,248-13,987)$ \\
\hline & Medio & 1 & 1,3 & 23 & 2,4 & & 1 \\
\hline \multirow{3}{*}{ Régimen de seguridad social } & Contributivo & 9 & 12,0 & 174 & 18,3 & 0,377 & $0,941(0,306-2,892)$ \\
\hline & Subsidiado & 60 & 80,0 & 678 & 71,3 & & $1,611(0,630-4,116)$ \\
\hline & No asegurado & 5 & 6,7 & 91 & 9,6 & & 1 \\
\hline \multirow{5}{*}{ Nivel de escolaridad } & Sin estudios & 5 & 6,7 & 58 & 6,1 & 0,126 & $1,207(0,220-6,611)$ \\
\hline & Primaria incompleta & 36 & 48,0 & 317 & 33,3 & & $1,590(0,364-6,952)$ \\
\hline & Secundaria & 29 & 38,7 & 490 & 51,5 & & $0,829(0,188-3,649)$ \\
\hline & Técnico/tecnólogo & 3 & 4,0 & 58 & 6,1 & & $0,724(0,114-4,583)$ \\
\hline & Universitaria & 2 & 2,7 & 28 & 2,9 & & 1 \\
\hline \multirow{5}{*}{ Ocupación } & Trabaja & 19 & 25,3 & 310 & 32,6 & 0,012 & $0,610(0,262-1,419)$ \\
\hline & Estudiante & 8 & 10,7 & 214 & 22,5 & & $2,039(0,776-5,363)$ \\
\hline & No trabaja ni busca trabajo & 6 & 8,0 & 48 & 5,0 & & $2,066(1,130-3,777)$ \\
\hline & Ama de casa & 29 & 38,7 & 229 & 24,1 & & $1,414(0,680-2,940)$ \\
\hline & Otra & 13 & 17,3 & 150 & 15,8 & & 1 \\
\hline \multirow{5}{*}{ Estado civil } & Soltero & 28 & 37,3 & 458 & 48,2 & 0,037 & 1 \\
\hline & Casado & 7 & 9,3 & 80 & 8,4 & & $1,431(0,605-3,388)$ \\
\hline & Unión libre & 29 & 38,7 & 340 & 35,8 & & $1,395(0,815-2,389)$ \\
\hline & Separado/divorciado & 6 & 8,0 & 56 & 5,9 & & $1,753(0,695-4,417)$ \\
\hline & Viudo & 5 & 6,7 & 17 & 1,8 & & $4,811(1,654-13,993)$ \\
\hline \multirow{3}{*}{ Ciudad } & Bogotá & 24 & 32,0 & 323 & 34,0 & 0,585 & 1 \\
\hline & Buenaventura & 29 & 38,7 & 313 & 32,9 & & $1,247(0,710-2,189)$ \\
\hline & Medellín & 22 & 29,3 & 315 & 33,1 & & $0,940(0,516-1,711)$ \\
\hline
\end{tabular}


Aunque no se presentó asociación estadística significativa, es importante resaltar que las personas que llevaron a cabo el desplazamiento de forma individual tienen una probabilidad más baja de presentar TEPT que quienes lo hicieron en forma grupal, ya sea con otros miembros de la familia o de la comunidad, es decir, cuando se lleva a cabo en forma masiva (OR=0,410 IC95 \% 0,147-1,142, $p=0,078$ ).

De acuerdo con los datos analizados un poco más de siete de cada 10 personas que han sufrido desplazamiento en la vida han presentado TEPT. La probabilidad de ser diagnosticado con TEPT es 1,3 veces mayor en las personas que tienen más de un año en situación de desplazamiento respecto a quienes tienen la misma situación hace menos de un año (OR=1,303 IC95 \% 0,512$3,322)$.

Con respecto al número de personas con las que se llevó a cabo el desplazamiento, seis de cada 10 (60,6 \%) de quienes presentaron TEPT lo hicieron en grupos pequeños, entre 1 y 5 personas; y de acuerdo con el actor que influyó en la salida del lugar de origen, es decir, en el desplazamiento, $58,7 \%$ expresó que fue la guerrilla y $33,3 \%$ los paramilitares. Aunque no se presenta evidencia estadística respecto a las anteriores asociaciones, resalta el hecho que cuando se da el desplazamiento masivo, es decir, con más de 10 personas, la probabilidad que se presente el TEPT es 1,8 veces mayor que cuando se desplazan con menos personas (OR= 1,879 IC95 \% 0,803 - 4,393, p= 0,248); en la misma línea, la probabilidad de presentar el TEPT al ser desplazado por el ejército es 2,3 veces mayor que si hubiese sido desplazado por otros actores (OR= 2,33 IC95 \% 0,208 - 26,225, $p=0,962$ ).

La única condición del desplazamiento que presenta una asociación estadísticamente significativa es la forma como se sale del lugar de residencia $(p=0,006)$, de modo que salir en el carro de algún conocido aumenta la probabilidad de la presencia de TEPT en casi tres veces con relación a salir en un transporte de servicio público. En la misma, línea salir caminando aumenta dicha probabilidad un poco más de dos veces. (OR= 2,721 IC95 \% 1,342 - 5,515; 2,179 IC95 \% $1,244-3,817, p=0,006)$ (ver Tabla 4). 
Tabla 4. Asociación entre condiciones del desplazamiento y el trastorno por estrés postraumático en la población de estudio ( $\mathrm{N}=1026)$.

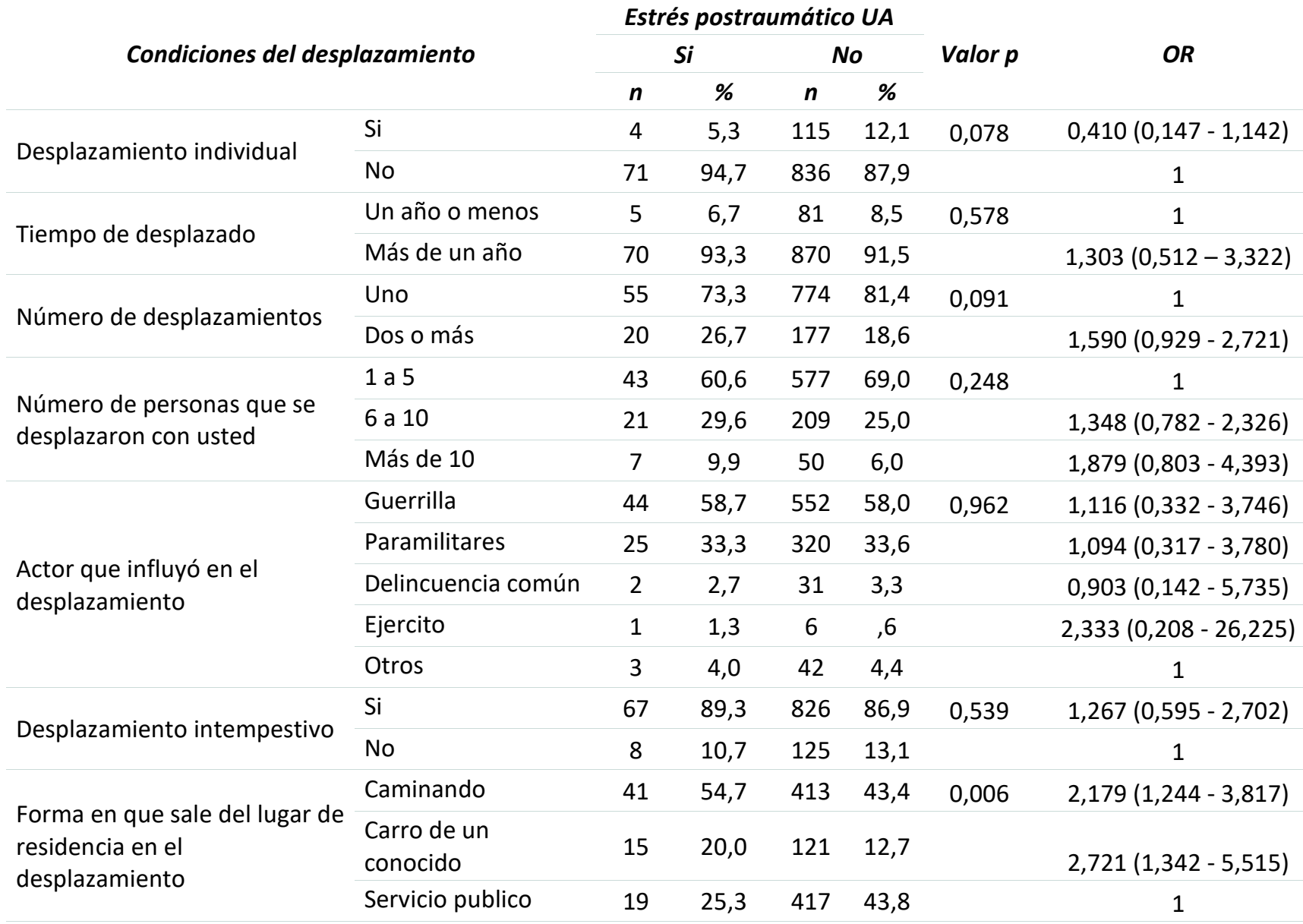

\section{Discusión}

Con el propósito de determinar la prevalencia del TEPT y los factores demográficos y condiciones del desplazamiento asociadas en población desplazada por la violencia producto del conflicto armado, en tres ciudades de Colombia: Bogotá, Medellín y Buenaventura, se analizaron los datos del estudio "Salud mental en víctimas de desplazamiento forzado por la violencia en Colombia. El caso de Bogotá, Medellín y Buenaventura” (Universidad CES, 2018). En el presente estudio se encontró una prevalencia de vida del TEPT en personas desplazadas en tres cuidades de Colombia del 9,9 \%, en el último año de 7,3\% y en el último mes de 4,4\%. La prevalencia de vida del TEPT registrada en el ámbito mundial es de 1 a $4 \%$, que puede variar con el evento estresante, la edad o el sexo, (Pérez-Olmos, Fernandez-Piñeres, \& RodadoFuentes, 2005); lo que conlleva a plantear que en los participantes de la presente investigación se duplica la presencia del TEPT como producto del desplazamiento forzado, el cual implica la 
salida de su territorio y, además, las múltiples pérdidas que van desde sus pertenencias físicas, hasta de sus seres queridos, relaciones y todo lo concerniente con el entorno sociocultural (Morales et al, 2009); de este modo se reconoce el desplazamiento como un evento que afecta de manera significativa la salud mental y por ende repercute en la salud pública de una localidad, región o país. La prevalencia de vida del TEPT de 9,9\% encontrada en el presente estudio, es similar a la prevalencia de entre 10\%-15\% reportada en el Estudio Nacional de Salud Mental y Colciencias (2015) en población desplazada. Este mismo estudio señala que el TEPT en población general presenta una prevalencia global en la vida entre $3 \%$ y $8 \%$; lo que apoya el planteamiento de Cudris-Torres y Barrios-Núñez (2018), quienes manifiestan que las personas que han experimentado un acontecimiento intenso amenazante tienen una mayor probabilidad de vivenciar el TEPT.

La presencia de TEPT en la población desplazada se evidenció independiente del sexo o la edad de las personas; además, puede manifestarse en cualquier momento de la vida después de la exposición al evento traumático. Las rupturas y pérdidas en todos los sentidos, económico, social, familiar y cultural, que implica el desplazamiento forzado no se olvidan fácilmente y se convierten en una carga para el resto de la vida; así, independientemente del lugar a donde se llegue y las nuevas condiciones de vida, la persona desplazada siempre tiene presente su pasado y la condición de vulnerabilidad dada por el desplazamiento, tal como afirman Martínez, Castro y Antivar (2020) "el desplazamiento trae consigo huellas indelebles, las mujeres sobrevivientes son estigmatizadas y sus saberes desvalorizados socialmente, además de las afectaciones emocionales, identitarias y sociales" (p. 278), y Morales, Muñoz y Acevedo (2012) "darse cuenta de que se está vivo pero sin nada de lo material, fruto del esfuerzo y del trabajo de años de las familias; el temor de ser perseguidos por los actores armados y el no saber que les depara el destino" (p. 88).

En el presente estudio solo se encontró asociación estadísticamente significativa entre TEPT y las variables de ocupación y estado civil; otros estudios como el de Pérez-Olmos et al. (2005), realizado en niños en contexto de guerra, no encontraron ninguna asociación con las variables sociodemográficas, y el de Echenique et al. (2008) reportó asociación con el barrio en el cual vivían las personas que fueron afectadas por el desplazamiento forzoso.

El estudio primario del cual emergen los datos de la presente investigación procuró mantener una distribución porcentual con respecto a cada ciudad (Medellín, Bogotá y Buenaventura) y a los grupos de edades, no obstante, el análisis de los datos refleja que las mujeres son las más afectadas $(53,5 \%)$ por el fenómeno del desplazamiento forzado; tal como lo han evidenciado 
Sinisterra, Figueroa, Moreno, Robayo y Sanguino (2010), quienes reportan un 65\% de mujeres víctimas del desplazamiento, y Londoño, Siachá y Gonzales (2011) que hallaron un 80 \%.

El desplazamiento impacta de manera diferentes a hombres y mujeres, pero son ellas la que tienden a desplazarse (Ferrel et al., 2020; Martínez et al., 2020) en tanto los hombres son los tienden a ser asesinados, desaparecidos o formar parte de las filas de los grupos armados. De este modo, en muchos casos las mujeres son las sobrevivientes de esta situación de conflicto, las que debe velar por las nuevas condiciones de vida y por la crianza de sus hijos, en medio de la incertidumbre y la pérdida, no solo de sus seres queridos, sino de sus pertenecías materiales, su cultura, sus raíces y ante todos de su contexto de vida, como lo evidencian Martínez et al. (2020), cuando afirman que las mujeres "se convierten en jefes del hogar a causa del abandono, desaparición y pérdida de sus parejas; sus dinámicas familiares se ven trastocadas, asumiendo la mayoría de los casos el rol de cuidadoras y proveedoras económica". (p.278).

Con respecto a la prevalencia del TEPT según el sexo, se evidenció que la mujer tiene mayor probabilidad de presentarlo en comparación con los hombres, resultado similar al de diversos estudios (Sinisterra et al., 2010); Torres et al., 2021; Londoño et al., 2011); sin embargo, difiere con el de Sarmiento (2016), quien encontró que los más afectados fueron los hombres. Asimismo, en la presente investigación se encontró que las personas entre 45 y 65 años de edad tienen más probabilidades de presentar TEPT en relación con los jóvenes, lo cual puede obedecer a la edad en la que se llevó a cabo el desplazamiento, de modo, que a menor edad menos consciencia de lo que estaba sucediendo (Morales et al., 2012). En la misma dirección, se encontró en este estudio que tiene mayor riesgo del TEPT los que solo han alcanzado estudios primarios y son viudos, en contraste con lo hallado por (Alejo et al. (2007) quienes plantean que son los universitarios y los solteros.

Además, según los datos analizados tener más de un año en situación de desplazamiento aumenta la probabilidad de presentar TEPT en 1,3 veces, Este resultado cobra importancia en cuanto la mayoría de la población estudiada (91,6\%) llevan más de un año en condición de desplazados. No obstante, Alejo et al. (2007) identificaron una asociación negativa del tiempo en situación de desplazamiento y la prevalencia de TEPT ( $r h o=-0.4, p<0.000$ ), es decir, que, a mayor tiempo de desplazamiento menor prevalencia de TEPT.

Los hallazgos presentados en esta investigación permiten una aproximación desde la perspectiva cuantitativa a la problemática del TEPT y del desplazamiento forzado en Colombia, contribuyendo de esta manera en el diseño de planes de prevención, promoción e intervención 
articulados con el campo de la salud pública. En la misma dirección, la determinación de las prevalencias de vida y año del TEPT se constituye en un importante insumo para el área de la investigación en salud mental puesto que permite hacer estudios comparativos.

Respecto a las limitaciones, esta investigación se realizó con fuentes secundarias, lo que conlleva asumir los posibles sesgos del estudio primario. Por otro lado, dado que es un estudio transversal no se puede atribuir relación de causalidad entre las variables sociodemográficas y las condiciones del desplazamiento con el TEPT.

\section{Conclusiones}

Las personas víctimas de desplazamiento forzado por la violencia en Colombia presentan una prevalencia del TEPT más elevada respecto a la población general.

Esta afectación a la salud mental se presenta independiente de las condiciones en que se llevó a cabo el desplazamiento, las características sociodemográficas, es decir, que afecta hombres, mujeres, niños y niñas, y en cualquier momento de la vida; por tanto, es muy importante hacer tamizajes periódicos en poblaciones que han sido víctimas de desplazamiento forzado con el fin de minimizar los efectos del TEPT y por ende de otras comorbilidades que se puedan presentar, garantizando así condiciones reales de reparación integral a las víctimas.

\section{Referencias}

Agencia de la ONU para los refugiados [ACNUR]. (2019). Tendencias globales. Desplazamiento forzado 2019. https://www.acnur.org/5eeaf5664.pdf

Achilli, J., Rodríguez, M., \& Folino, J. (2014). Postraumatic mental disorders in traders victims of crime. Vertex, 25(118), 405-412. https://pubmed.ncbi.nlm.nih.gov/26098818/

Alejo, E. G., Rueda, G., Ortega, M., \& Orozco, L. C. (2007). Estudio epidemiológico del trastorno por estrés postraumático en población desplazada por la violencia política en Colombia.

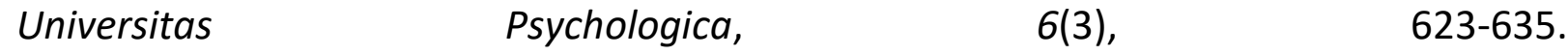
https://revistas.javeriana.edu.co/index.php/revPsycho/article/view/146

Bados, A. (2015). Trastorno por estrés postraumático. Universidad de Barcelona.

Beck, J. G., \& Sloan, D. M. (2012). The Oxford Handbook of Traumatic Stress Disorders (1.a ed.). Oxford University Press. https://doi.org/10.1093/oxfordhb/9780195399066.001.0001

Campo-Arias, A., Oviedo, H. C., \& Herazo, E. (2014). Prevalencia de síntomas, posibles casos y trastornos mentales en víctimas del conflicto armado interno en situación de desplazamiento en Colombia: Una revisión sistemática. Revista Colombiana de Psiquiatría, 43(4), 177-185. https://doi.org/10.1016/i.rcp.2014.07.003 
Cudris-Torres, L., \& Barrios-Núñez, A. (2018). Malestar psicológico en víctimas del conflicto armado. Revista CS, (26), 75-90. https://doi.org/10.18046/recs.i25.2654

Diaz, L., \& Serrano, C. (2016). Exposición al trauma: Trastorno de estrés postraumático (TEPT) en víctimas del conflicto armado colombiano y posibles retos del programa de atención psicosocial y salud integral a víctimas (PAPSIVI). Revista Cambios y Permanencias 7, 801814. https://revistas.uis.edu.co/index.php/revistacyp/article/view/7075

Echenique, C., Medina, L. M., Medina, A. R., \& Ramírez, A. (2008). Prevalencia del trastorno por estrés postraumático en población desplazada por violencia, en proceso de reestablecimiento en Sincelejo. Psicol desde El Caribe, 21, 125-135. https://rcientificas.uninorte.edu.co/index.php/psicologia/article/view/1644/5330

Ehlers, A., Hackmann, A., \& Michael, T. (2004). Intrusive re-experiencing in post-traumatic stress disorder: Phenomenology, theory, and therapy. Memory, 12(4), 403-415. https://doi.org/10.1080/09658210444000025

Ferrel, F. R., Ferrel, L. F., Cañas-Herazo, C. V., Barros, M.F., \& Yáñez, H. (2020). Estilo de vida y estrés postraumático en mujeres desplazadas víctimas de abuso sexual en Santa Marta, Colombia. Revista Cubana de Salud Pública, 46(1), e1363. http://www.revsaludpublica.sld.cu/index.php/spu/article/view/1363

Londoño Calle, N., Sicachá Espinosa, M. A., \& Gonzales Sánchez, J. C. (2011). Posibles manifestaciones del trastorno por estrés post-traumático (TEPT) en adultos desplazados por el conflicto armado del asentamiento "Acacias bajo" en Armenia- Quindío. Sinapsis, 3(3), 172-185.

Martínez Chaparro, A.M., Castro Yepes, L.M., \& Antivar Londoño, D. F. (2020). Apoyo social en mujeres sobrevivientes de desplazamiento intraurbano en Medellín-Colombia. Revista de Paz y Conflicto, 13(1), 275-291. http://dx.doi.org/10.30827/revpaz.v13i1.9591

Ministerio de Salud y Protección Social, \& Colciencias. (2015). Encuesta Nacional de Salud Mental. Tomo http://www.odc.gov.co/Portals/1/publicaciones/pdf/consumo/estudios/nacionales/CO 031102015-salud mental tomol.pdf

Morales Mesa, S. A., Muñoz Arroyave, C. O., Ghiso Cotos, A. M., Tobón Hoyos, J.F., \& Patiño Muñoz, M. (2009). Desplazamiento de poblaciones: Un acercamiento contextual y teórico. Fondo Editorial Luis Amigó - FIUC. https://www.funlam.edu.co/modules/fondoeditorial/item.php?itemid=154

Morales Mesa, S. A., Muñoz Arroyave, C. O., \& Acevedo Valencia, J. M. (2012). Trayectorias de socialización de la niñez desplazada por la violencia en los asentamientos de la ciudad de Medellín. Fondo Editorial Luis Amigó - FIUC. https://www.funlam.edu.co/modules/fondoeditorial/item.php?itemid=186

Ozer, E. J., Best, S. R., Lipsey, T. L., \& Weiss, D. S. (2003). Predictors of posttraumatic stress disorder and symptoms in adults: A meta-analysis. Psychological Bulletin, 129(1), 52-73. https://doi.org/10.1037/0033-2909.129.1.52 
Pérez-Olmos, I, Fernández-Piñeres, P. E., \& Rodado-Fuentes, S. (2005). Prevalencia del Trastorno por Estrés Postraumático por la Guerra, en Niños de Cundinamarca, Colombia. Rev. Salud Pública, 7(3), 268-280. https://scielosp.org/pdf/rsap/2005.v7n3/268-280/es

Red Nacional de Información [RNI]. (2020). Desplazamiento-personas. Datos Nacional y departmental. https://cifras.unidadvictimas.gov.co/Home/Desplazamiento

Sarmiento, R. (2016). Trastorno de estrés postraumático, ansiedad y depresión en adolescentes y adultos expuestos al conflicto armado en Colombia 2005-2008. Medicina, 2(113), 134156. https://revistamedicina.net/ojsanm/index.php/Medicina/article/view/113-4

Sinisterra Mosquera, M., Figueroa Lozano, F., Moreno Gutiérrez, V., Robayo, F., \& Sanguino Leal, J. (2010). Prevalencia del trastorno de estrés post traumático en población en situación de desplazamiento en la localidad de Ciudad Bolívar, Bogotá, Colombia 2007.

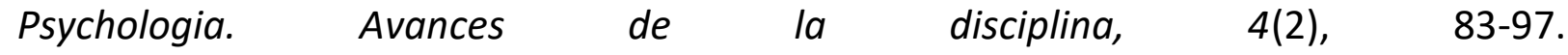
http://www.scielo.org.co/scielo.php?script=sci arttext\&pid=S190023862010000200008\&lng=en\&nrm=iso\&tlng=es

Ministerio de la Protección Social de Colombia, Oficina de las Naciones Unidas contra la Droga y el Delito [UNODC], \& Universidad CES. (2010). Situación de salud mental del adolescente, Estudio Nacional de Salud Mental, Colombia, The WHO World Mental Health Survey Consortium. http://psicopediahoy.s3.amazonaws.com/saludmentaladolescentecolombia.pdf

Torres-Salazar, Y.M., Mejía-Jaimes, L., Conde-Cotes, C.A. \& Botelho-De Oliveira, S. (2021). Víctimas del desplazamiento forzado: comorbilidad entre trastorno por estrés postraumático (TEPT) y depresivo mayor (TDM). Informes Psicológicos, 21(1), 133-149 http://dx.doi.org/10.18566/infpsic.v21n1a09

Universidad CES. (2018). Salud mental en víctimas de desplazamiento forzado por la violencia en Colombia. El caso de Bogotá, Medellín y Buenaventura. Medellín: Universidad CES. 